\title{
Pose Angular-Aiding for Maneuvering Target Tracking
}

\author{
Chun Yang \\ Sigtem Technology, Inc. \\ 113 Clover Hill Lane \\ Harleysville, PA 19438 \\ chunyang@sigtem.com
}

\author{
Erik Blasch \\ Air Force Research Lab/SNAA \\ 2241 Avionics Circle \\ WPAFB, OH 45433 \\ erik.blasch@wpafb.af.mil
}

\begin{abstract}
With high-resolution radar sensors such as HRRR and SAR, ground targets become visible more as a rich set of radar signatures corresponding to the target geometrical details than as a single reflector with an equivalent RCS. This has enabled target classification and identification (ID). As a "by-product" of the target ID process, the pose angles are made available. For ground targets, which are constrained to move on the earth surface, their velocity vector direction is aligned most of time along the body's longitudinal axis. As a result, the pose angles carry kinematic information that can be used to aid target tracking particularly during the maneuvering periods. In this paper, we investigate the potential benefits of pose angular aiding and the requirements on pose angular measurements in order to realize such benefits. Simulation results are presented to illustrate the concept and performance.
\end{abstract}

Keywords: Feature Aiding, Tracking, Pose, IMM

\section{Introduction}

Feature-aided tracking remains to be an area of active search, to support which a public-domain database called "Feature-Aided Tracking of Stop-move Objects (FATSO)" has been generated by the Sensor Directorate of the Air Force Research Laboratory (AFRL/SN) [1]. Compared to conventional tracking with post-detection position observables (i.e., range and bearing), feature-aided tracking (FAT) expands at least in two aspects:

- Low-level measurements, which are compared with reference templates in a database for match. When successful, it provides target type, viewing angles (or pose angles), and other information.

- Extended target state, which will include not only the kinematic variables (i.e., position, velocity, and possibly acceleration) but also target's orientation relative to its environment (terrain and road).

For a high range resolution radar (HRRR), the lowlevel measurements are in the form of range profiles as shown in Figure 1. The range profile is a onedimensional measurement of target radar reflectivity along the radar to target line of sight (LOS) vector where the amplitudes are statistical features. This look vector, when expressed in the target body frame in terms of the aspect (or articulation) and depression angles, is called a "pose" as illustrated in Figure 2. For practical reasons, a target is pre-sampled into a template library in its range profile at discrete poses. A successful template matching therefore identifies the target type and at the same time produces the pose at which the range profile is viewed.

Most target tracking and ID systems are implemented independently. This has both theoretical and practical reasons. One practical limitation in the past was the lack of sensor accuracy/resolution and powerful computers for reliable implementation in

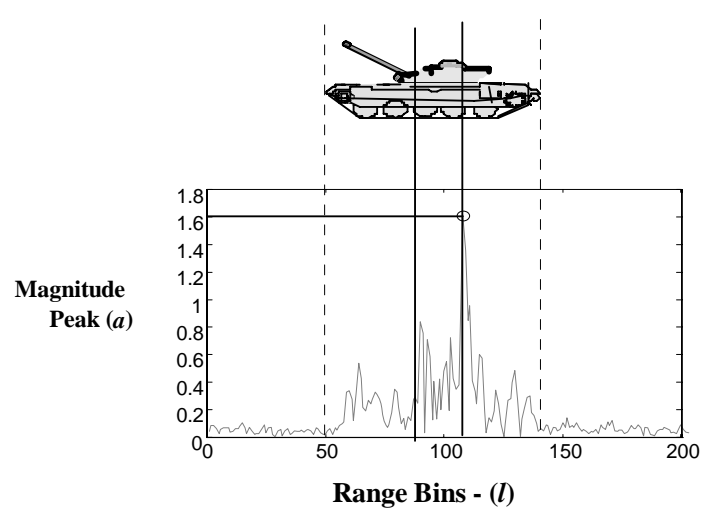

Figure 1. High Resolution Range Profile

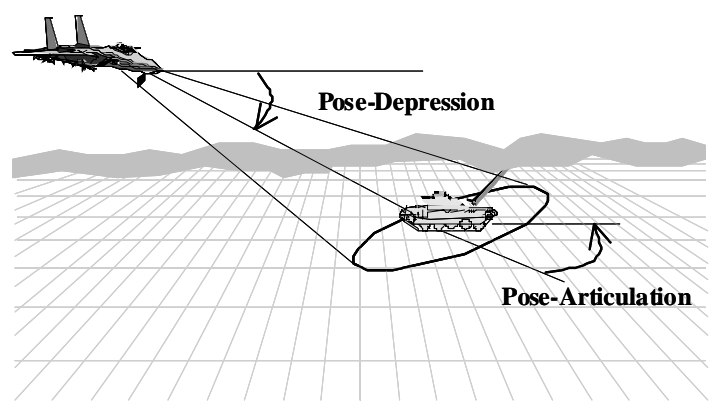

Figure 2. Pose Angle Defined in Terms of Aspect (Articulation) and Depression Angles 
real time. When target tracking and ID are considered jointly, we deal with a hybrid space. The target kinematic state and its measurements are continuousvalued whereas the target type is discrete-valued and so is the target pose due to quantization.

In our previous work [2; 3], we have identified possible couplings between tracking and ID systems via pose, kinematic, and association constraints that can be exploited to improve performance.

In this paper, we continue our early efforts and will investigate the potential benefits of pose angular aiding. Target pose angles are made as part of the target ID process. For ground targets, they are constrained to move on the earth surface and more importantly their velocity vector direction is aligned most of time along the body's longitudinal axis. As a result, the pose angles carry kinematic information that can be used to aid target tracking particularly during the maneuvering periods. It can be used either as a maneuver indicator or as an extra measurement, both helping maneuvering target tracking. In addition, we will determine the requirements on pose angular measurements (accuracy and latency) in order to realize such benefits. Simulation results are presented to illustrate the concept and performance.

\section{Pose Angular Features}

Target pose is the viewing direction expressed in the target body coordinates. It is the same as the LOS vector, which will be denoted by $\underline{\underline{l}}^{\text {[a] }}$ when measured in the radar frame and by $\underline{\underline{l}}^{\mathrm{b}]}$ when seen in the target frame. They are related by:

$$
\begin{aligned}
& \underline{\underline{l}}^{\mathrm{b}]}=\mathrm{L}_{\mathrm{a}}{ }^{\mathrm{b}} \underline{\underline{l}}^{[\mathrm{a}]} \\
& \mathrm{L}_{\mathrm{a}}^{\mathrm{b}}=\mathrm{L}_{\mathrm{r}}{ }^{\mathrm{b}} \mathrm{L}_{\mathrm{g} 2}{ }^{\mathrm{r}} \mathrm{L}^{\mathrm{g}}\left(\mathrm{L}_{\mathrm{i}}^{\mathrm{a}}\right)^{\mathrm{T}}
\end{aligned}
$$

where $\mathrm{L}_{\mathrm{a}}{ }^{\mathrm{b}}$ is the rotation matrix from the airborne sensor frame to the target body frame.

Conceptually, it is constructed from the following rotations: (1) $\mathrm{L}_{\mathrm{i}}{ }^{\mathrm{a}}$ is the attitude of the aircraft sensor frame to the common reference frame (roll, pitch and yaw angles) provided by the onboard inertial navigation system (INS), (2) $\mathrm{L}_{\mathrm{i}}^{\mathrm{g}}$ is the rotation matrix from the common reference frame to the terrain surface frame, (3) $\mathrm{L}_{\mathrm{g}}{ }^{\mathrm{r}}$ is the rotation matrix from the terrain surface to the road segment with an additional azimuth angle, and (4) $\mathrm{L}_{\mathrm{r}}^{\mathrm{b}}$ is the rotation matrix from the road segment to the target vehicle with an additional azimuth angle.

The rotation matrix from the common reference frame to the terrain surface frame, $\mathrm{L}_{\mathrm{i} 2 \mathrm{~g}}$, is related to the local terrain surface orientation angles, which are functions of the target location. The rotation matrix from the terrain surface frame to the target vehicle body frame $\mathrm{L}_{\mathrm{g}}{ }^{\mathrm{b}}=\mathrm{L}_{\mathrm{r}}{ }^{\mathrm{b}} \mathrm{L}_{\mathrm{g}}{ }^{\mathrm{r}}$ can be approximately given by the direction of velocity vector.

To illustrate, consider two simple cases in Figure 3. The first case in Figure 3(a) is a tail chase where the target and sensor are in the same vertical plane. When the airborne sensor is level, the depression angle $\mu$ is related to the elevation angle of the radar $\eta$ and the terrain surface slope $\Theta$ by:

$$
\mu=\eta+\Theta
$$

The second case as shown in Figure 3(b) is on a flat earth where the airborne sensor is also level. The aspect angle $\varphi$ is then related to the azimuth angle of the radar $\xi$, the road direction $\psi$, and the angular offset of the velocity vector relative to the $\operatorname{road} \beta$ as:

$$
\varphi=180^{\circ}-\xi-\psi-\beta
$$

For the second case, assume that the velocity direction is in alignment with the pose aspect angle (i.e., without sideslip). Then the pose aspect angle can be used as an additional measurement [4]:

$$
\varphi=\tan ^{-1} \frac{v_{y}}{v_{x}}
$$

where $v_{x}$ and $v_{y}$ are the velocity components in the $x$ and y-axis directions, respectively.

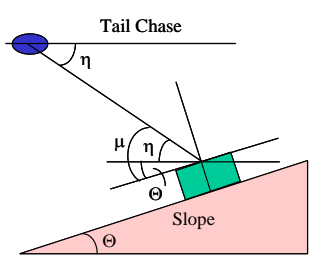

(a)

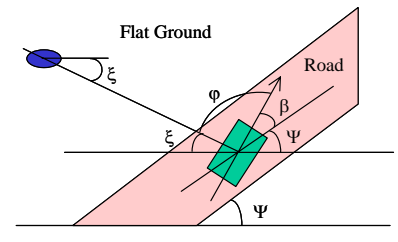

(b)
Figure 3 - Pose Angles Related to Radar and Terrain/Road Conditions

If we use the extended Kalman filter, this nonlinear measurement will be linearized around the predicted value of the two velocity components. The prediction error, denoted by $\Delta \varphi$, is related to the target velocity as:

$$
\begin{aligned}
& \Delta \varphi=\varphi-\tan ^{-1} \frac{\bar{v}_{y}}{\bar{v}_{x}} \\
& =\left[\begin{array}{ll}
\frac{-\bar{v}_{y}}{\bar{v}_{x}^{2}+\bar{v}_{y}^{2}} & \frac{\bar{v}_{x}}{\bar{v}_{x}^{2}+\bar{v}_{y}^{2}}
\end{array}\right]\left[\begin{array}{c}
v_{x} \\
v_{y}
\end{array}\right]
\end{aligned}
$$

where the quantities with an over bar are the predicted values.

This pose-derived measurement can be used to complement the range and bearing measurements. 


\section{Simulation Scenario}

We consider a simple scenario in which a target maneuvers on an $\mathrm{x}-\mathrm{y}$ plane. The target is measured every $\mathrm{T}=10$ seconds. At $t=410 \mathrm{~s}$, it starts a slow 90 degrees turn, which is completed in 190 seconds. A second, fast, 90 degrees turn starts at $t=610 \mathrm{~s}$ and is completed in 40 seconds.

The target state vector is chosen as $\underline{X}=[x \dot{x} y \dot{y}]^{\mathrm{T}}$ with its initial values $\underline{X}(0)=[2000 \mathrm{~m}, 0 \mathrm{~m} / \mathrm{s}, 10000 \mathrm{~m}$, $15 \mathrm{~m} / \mathrm{s}]^{\mathrm{T}}$. The slow turn is the result of acceleration inputs $a_{x}=a_{y}=0.075 \mathrm{~m} / \mathrm{s}^{2}, 410 \mathrm{~s} \leq t \leq 610 \mathrm{~s}$ and the fast turn is due to inputs $a_{X}=a_{y}=-0.3 \mathrm{~m} / \mathrm{s}^{2}, 610 \mathrm{~s} \leq t$ $\leq 650$ s.

Without maneuver, the target is modeled (separately in $x$ and $y$ coordinates) by:

$$
\underline{X}(k+1)=\left[\begin{array}{cc}
1 & T \\
0 & 1
\end{array}\right] \underline{X}(k)+\left[\begin{array}{c}
\frac{T^{2}}{2} \\
\mathrm{~T}
\end{array}\right] v(k)
$$

where $v(\mathrm{k}) \sim \mathrm{N}(0, \mathrm{Q})$. The measurement model is:

$$
\underline{Z}(k)=\left[\begin{array}{ll}
1 & 0
\end{array}\right] \underline{X}(k)+w(k)
$$

where $\mathrm{w}(\mathrm{k}) \sim \mathrm{N}\{0, \mathrm{R}\}$.

The model transition probability matrix is defined as:

$$
\Pi=\left[\begin{array}{lll}
0.8 & 0.1 & 0.1 \\
0.1 & 0.8 & 0.1 \\
0.1 & 0.1 & 0.8
\end{array}\right]
$$

It is easy to see that the slow turn rate is $0.4478^{\circ}$ /sec while the fast turn rate is $2.1951^{\circ} / \mathrm{sec}$. For each sample interval of $10 \mathrm{~s}$, the angular increment is $4.478^{\circ} /$ sample and $21.951^{\circ} /$ sample, respectively. In the feature-aided tracking, the accuracy of pose angular measurements will be made commensurate with these numbers.

\section{Pose Angles As Maneuver Indicator}

For the analysis presented in this section, we assume that the target orientation (i.e., pose angles) is derived from a template matching process using the target's HRRR range profiles or SAR imagery patches with a database. Without sideslip angle, the vehicle orientation is aligned with the velocity vector. As a result, the estimated vehicle orientation in the local coordinate can provide an estimate of the direction of the target velocity vector. It can be used to help the tracker as a maneuver indicator (studied in this section) or an extra measurement (in Section 5).

Conventional tracking algorithms such as the IMM estimator [5] make use of positional measurements (range and bearing) alone. However, the change in velocity is faster than position after a turning maneuver for instance because position is integrated out of velocity. As a result, the use of change in orientation has the potential to reduce transient errors thanks to early and better estimation of the correct target model [6].

To appreciate this, let us compare the ideal angular difference between two consecutive pose articulation angles (i.e., an estimate of angular rate) and that calculated from the IMM velocity estimates. As shown in Figure 4, the IMM estimator lags the actual pose angular changes. These are places where the velocity errors develop in the filter estimates and the position errors follow suite.

Figure 5 shows the angular rates calculated from pose angular measurements when it is subject to white Gaussian errors with $\sigma_{\psi}=0.5^{\circ}, 1^{\circ}$, and $3^{\circ}$, respectively. The corresponding angular rate standard deviations are $\sigma_{\dot{\psi}}=0.707,1.414$, and $4.243^{\circ} /$ sample, respectively.

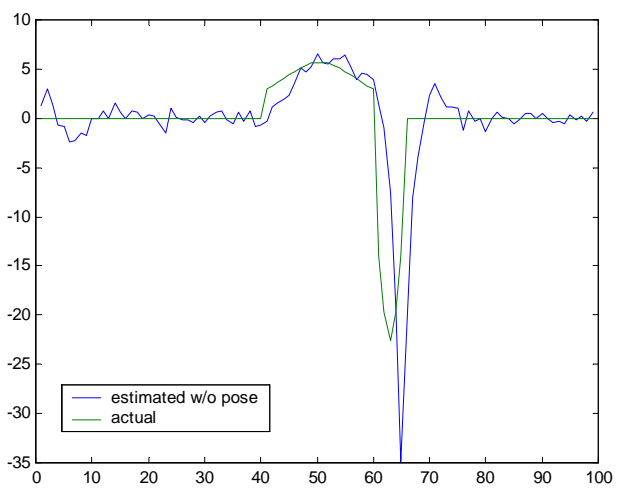

Figure 4. Latency in Angular Rate Estimation by Position-Alone IMM after Turns

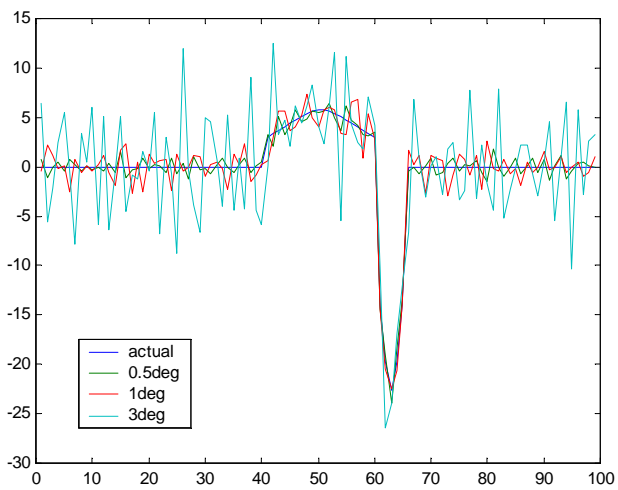

Figure 5. Angular Rate Measurements with Different Noise Levels

It is clear that filtering is needed to smooth out the noise and to provide a decision as to if a turn has occurred and if so in which direction and at which rate. Two estimation techniques are developed below. 
One is an IMM estimator for the pose articulation angle, which estimates the underlying angular rate and their model probabilities. The other is a Bayesian-Markov technique. Both are described below and evaluated with simulations.

\subsection{Bayesian-Markov Estimator of Turning Maneuvers}

By inspecting Figures 4 and 5, it is reasonable to believe that a consistent bigger than $3^{\circ} /$ sample change in angular rates indicates a maneuver turn. A Bayesian-Markov estimation algorithm can therefore be used to detect jumps (pose angular rate) in noise measurements (pose angle).

In this estimation technique, the abrupt change of heading is assumed to take place within a finite set of possibilities (mode), denoted by $\mathrm{m}(\mathrm{t}) \in \mathcal{M}=$ $\{1,2, \ldots, M\}$ with its indicator vector denoted by $\phi(t)$ such that $\phi_{\mathrm{i}}(\mathrm{t})=1$ if $\mathrm{m}(\mathrm{t})=\mathrm{i}$.

The behavior of $\mathrm{m}(\mathrm{t})$ is modeled by a Markov chain with the transition probability $\mathrm{Q}=\left[\mathrm{q}_{\mathrm{ij}}\right]$ defined as:

$$
\begin{aligned}
& \mathrm{q}_{\mathrm{ij}}=\mathrm{P}\{\mathrm{m}(\mathrm{t}+1)=\mathrm{j} \mid \mathrm{m}(\mathrm{t})=\mathrm{i}\}=\mathrm{P}\left\{\phi_{\mathrm{j}}(\mathrm{t})=1 \mid \phi_{\mathrm{i}}(\mathrm{t})=1\right\} \\
& \text { with } \sum_{j=1}^{M} q_{i j}=1
\end{aligned}
$$

The angular rate measurements can be modeled as:

$$
\begin{aligned}
z(t)=A[t, m(t)] z(t-1)+H[t, m(t)] \\
+G[t, m(t)] w(t)
\end{aligned}
$$

where the matrices $A(n \times n), H(n \times 1)$, and $G(n \times p)$ depend on the current mode $\mathrm{m}(\mathrm{t})$, and $\mathrm{w}(\mathrm{t})$ is an independent unit-variance white Gaussian process. The observation $\mathrm{z}(\mathrm{t})$ can be considered as a stochastic process with its mean and moment functions modulated by the jump mode.

Denote the measurement history by $\mathrm{Z}^{\mathrm{t}}=\{\mathrm{z}(\mathrm{s})$, $\mathrm{s} \leq \mathrm{t}\}$. The estimate of the jump mode $\phi(t)$ in the mean square sense given the current and past pose measurements $\mathrm{Z}^{\mathrm{t}}$ is written as:

$$
\hat{\phi}(t \mid t)=E\left\{\phi(t) \mid Z^{t}\right\}
$$

As the conditional expectation, it affords a natural interpretation that its $\mathrm{i}^{\text {th }}$ component is the a posteriori probability of $\phi_{i}(t)=1$ (the $\mathrm{i}^{\text {th }}$ state is true) given $Z^{t}$.

Then applying the Bayes' formula, we obtain a recursive algorithm to calculate the composite state estimate in which

$$
\hat{\phi}(t \mid t)=\frac{\operatorname{diag}\{L[z(t)]\} \hat{\phi}(t \mid t-1)}{L[z(t)]^{\prime} \hat{\phi}(t \mid t-1)}
$$

is the measurement updating equation;

$$
\hat{\phi}(t \mid t-1)=Q^{\prime} \hat{\phi}(t-1 \mid t-1)
$$

is the one step ahead prediction equation;

$$
L[z(t)]=\left[f _ { 1 } \left(z(t), f_{2}\left(z(t), \ldots, f_{M}(z(t)]\right.\right.\right.
$$

is the set of likelihood functions defined by

$$
\mathrm{f}_{\mathrm{i}}\left(\mathrm{z}(\mathrm{t})=\mathrm{N}\left\{\mathrm{z}(\mathrm{t}) ; \mathrm{A}_{\mathrm{i}} \mathrm{z}(\mathrm{t}-1)+\mathrm{H}_{\mathrm{i}}, \mathrm{G}_{\mathrm{i}} \mathrm{G}_{\mathrm{i}}{ }^{\prime}\right\}\right.
$$

and $\hat{\phi}(0 \mid 0)=\hat{\phi}_{0}$ is the initial condition.

Other techniques can be used to construct the mode filter including the point-process mode filter [7] and the confidence belief measures [8].

We present the simulation results next. In the present case, we have $\mathrm{M}=3, \mathrm{H}_{1}=0^{\circ}$ /sample; $\mathrm{H}_{2}=$ $5 \%$ sample, $\mathrm{H}_{3}=-5^{\circ}$ sample; $\mathrm{A}_{\mathrm{i}}=0$; and $\mathrm{G}_{\mathrm{i}}=$ $1 \%$ sample, for $\mathrm{i}=1,2$, 3 . In the Bayesian-Markov algorithm of Eq. (11), we use the same transition probability matrix as the IMM algorithm of Eq. (7).

Figure 6 shows the model probabilities when the angular error standard deviation is $0.5^{\circ}$, from which the angular difference is calculated as the measurements as in Eq. (9). Compared to position measurements alone, the use of pose articulation angles can obtain faster and more reliable indication of the most likely model of maneuvering than the

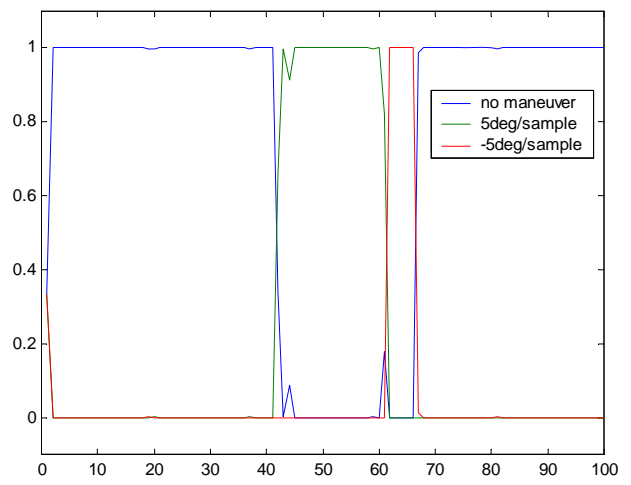

position data alone.

Figure 6. Model Probabilities from Pose Measurements with Low Noise

When the angular error standard deviation is increased to $1^{\circ}$, Figure 7 shows the model probabilities. There are some false transition trends but overall it provides faster indication of the most likely model.

When the angular error standard deviation is further increased to $3^{\circ}$, the model probabilities are not as reliable as the two previous cases because this level of angular noise is as large as the turn rate itself. Although it can indicate the true turns quickly, there are many false transition spikes. Clearly, the pose angular measurement accuracy plays a key role and this analysis imposes the performance requirements on the pose angular estimation algorithms. 


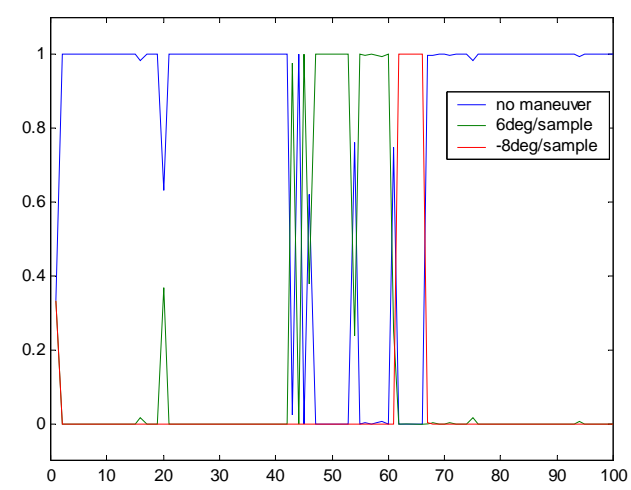

Figure 7. Model Probabilities from Pose Measurements with High Noise

If we work with angular measurements directly rather than their time-differences, we can employ the IMM algorithm and are expected to see noise reduction as presented below.

\subsection{IMM Estimator for Pose Angles}

For a target maintaining a constant heading, its orientation (i.e., the velocity vector) relative to the local reference frame also remains constant until it starts making turns. As a result, the change in heading as measured from the pose angles as shown in Figure 4 can be used to detect the underlying maneuvers.

A turning maneuver, as seen from the angular measurements, appears as a constant drift in one direction or the other. However, the drift rate (i.e., the angular turning rate) is unknown. In the IMM algorithm, this unknown is hypothesized as one of possible rate values.

It suffices to use a first-order model to relate the observed pose articulation angle $\psi_{\mathrm{k}}$ to the unknown turning rate $\omega_{\mathrm{k}}$ by:

$$
\psi_{k+1}=\psi_{k}+T \omega_{k}+v_{k}
$$

where $\mathrm{v}_{\mathrm{k}}$ is the process noise assumed to be independent zero-mean white Gaussian, and $\mathrm{T}$ is the sampling interval.

The measurement equation is given by:

$$
\breve{\psi}_{k}=\psi_{k}+w_{k}
$$

where $\mathrm{w}_{\mathrm{k}}$ is the measurement noise assumed to be independent zero-mean white Gaussian.

In the first simulation, three models are chosen for the unknown angular rate as $\Omega=[0,0.2865,-0.2865]$ $\left({ }^{\circ} / \mathrm{sec}\right)=[0,0.005,-0.005](\mathrm{rad} / \mathrm{sec})$, respectively. The angular measurement noise standard deviation is $\sigma_{\mathrm{w}}=0.5^{\circ}$. The process noise standard deviations are $\sigma_{\mathrm{v}}=\left[0.0001^{\circ}, 0.001^{\circ}\right.$ and $\left.0.001^{\circ}\right]$ for the three models, respectively. Figure 8 shows the model probabilities. The IMM filter rapidly and reliably captures the two turns. However, although in favor of, it is less certain when the vehicle goes straight. This is because the three models are very close in values.

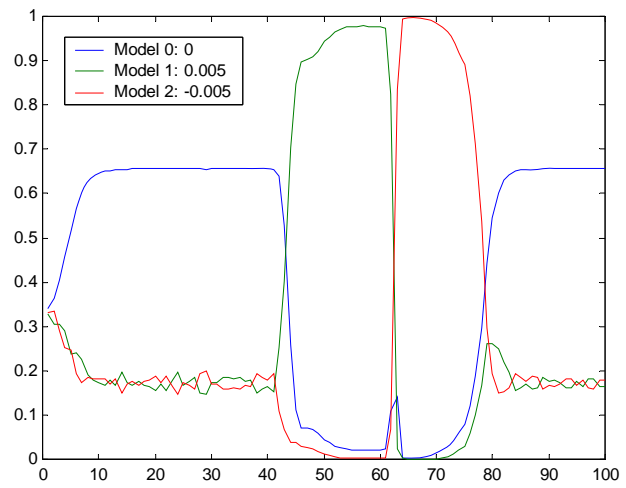

Figure 8. Model Probabilities with Closely Spaced Models

In the second simulation, all the parameters remain the same except for the angular rates that are chosen this time with larger separation as $\Omega=[0,0.5,-0.5]$ $(\% / \mathrm{sec})=[0,0.0087-0.0087](\mathrm{rad} / \mathrm{sec})$, respectively. Figure 9 shows the model probabilities where the IMM filter improves its probability for no maneuver model.

Figure 9. Model Probabilities with Larger Model

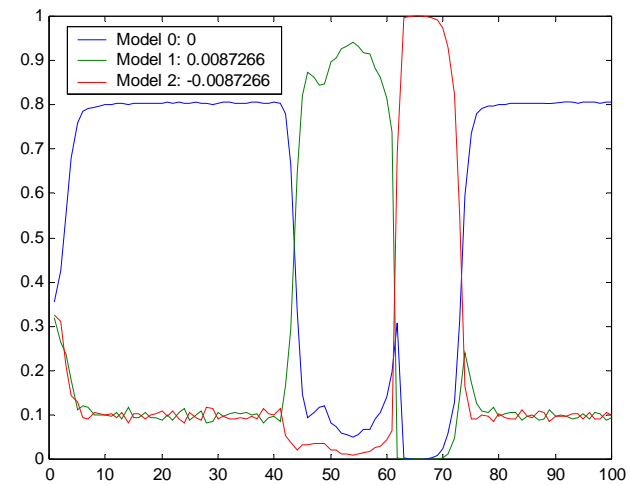

Separations

Figures 10 and 11 show two cases where the angular measurements are subject to large errors $\sigma_{\mathrm{w}}=$ $1^{\circ}$ and $3^{\circ}$, respectively. Although the IMM filter is less sure about its model when the vehicle goes straight, it is still in favor of the no maneuver model consistently. It is remarkable that with such large measurement errors, the filter can correctly determine the maneuver models. This is advantageous as compared to Figure 7 in the large noise case. 


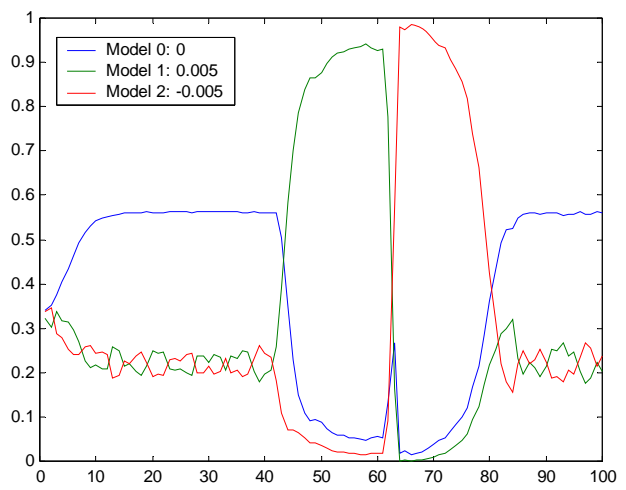

Figure 10 - Model Probabilities with Larger Measurement Errors $\left(1^{\circ}\right)$

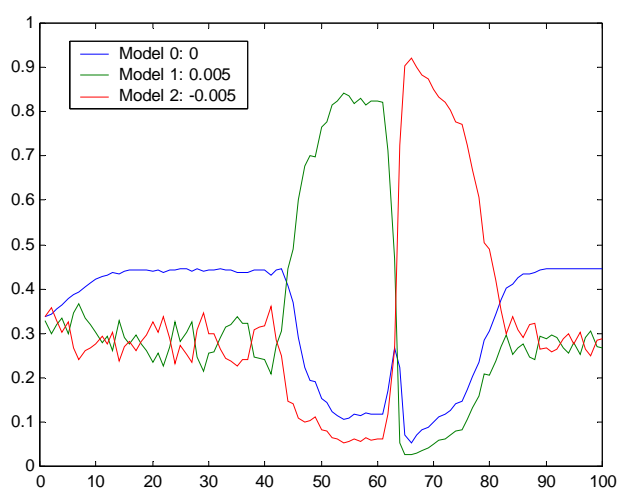

Figure 11 - Model Probabilities with Larger Measurement Errors $\left(3^{\circ}\right)$

\section{Pose Angles As Extra Measurements}

In this section, we study through simulation the use of the pose angular measurements as given in Eq. (4) to assist the IMM estimator. The position and pose angular measurement error standard deviations are $50 \mathrm{~m}$ and $1^{\circ}$, respectively. The acceleration models are $A_{\text {filter }}=[0,0.075,-0.3]\left(\mathrm{m} / \mathrm{s}^{2}\right)$ with the process noise variances being $\mathrm{Q}_{\text {filter }}=\left[0.01^{2}, 0.1^{2}, 0.1^{2}\right]$.

Figure 12 shows the measured and estimated trajectory in comparison to the actual one. Figures 13 and 14 show the sample history of the measured and estimated (IMM combined) position errors in the $\mathrm{x}$ and y-direction, respectively.

Figures 15 and 16 show the sample history of the actual, the individual model-estimated, and the combined velocity in the $\mathrm{x}^{-}$and $\mathrm{y}$-direction, respectively. Figure 17 shows the model probabilities by the IMM filter when the pose angular measurement is used, significant improvement over the cases without pose measurement.
Position and velocity estimation error statistics data

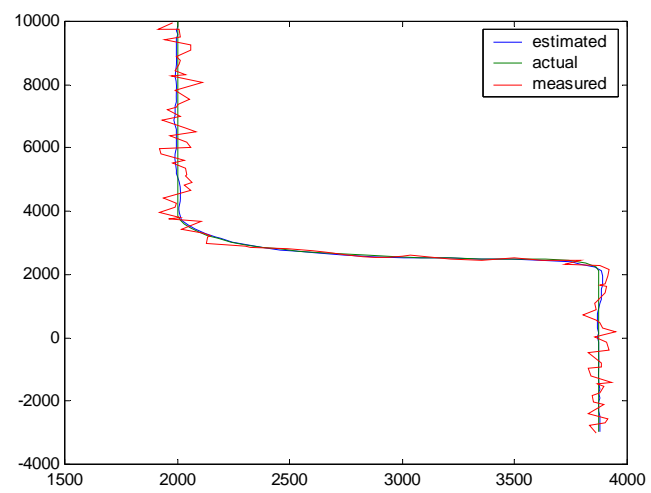

are shown in Table 1 for the cases where the angular

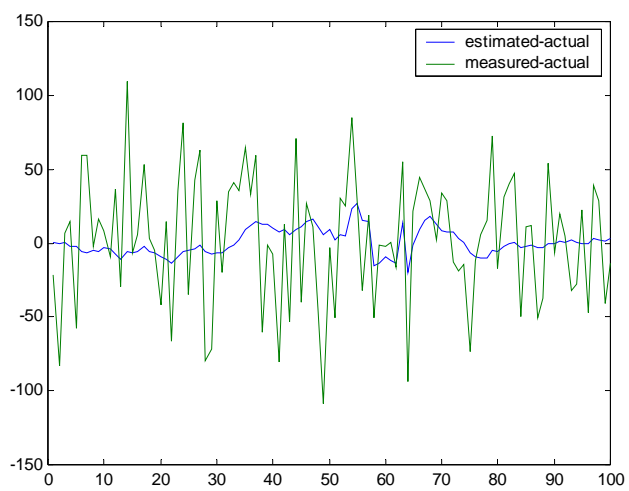

Figure 12. Target Trajectory

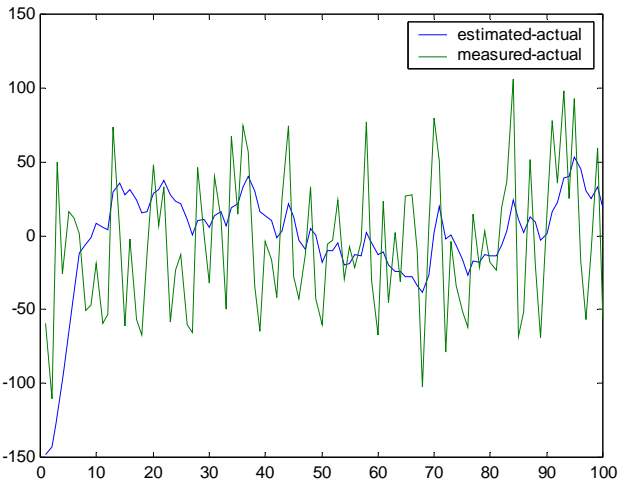

Figure 13. Position Errors (X)

Figure 14. Position Errors (Y)

measurement error standard deviation is $1^{\circ}$ and $2^{\circ}$ while the position measurement error standard deviation is $50 \mathrm{~m}$.

Table 1. Position and Velocity Estimation Errors Statistics

\begin{tabular}{|c|c|c|c|c|c|}
\hline Pose & STD & RMS-X & RMS-Y & RMS-VX & RMS-VY \\
\hline Without & $\sigma_{x, y}=50 \mathrm{~m}$ & 23.2785 & 31.1022 & 4.9666 & 5.3763 \\
\hline \multirow{2}{*}{ With } & $\sigma_{\psi}=1^{\circ}$ & 7.0033 & 22.2635 & 0.1405 & 0.5309 \\
\cline { 2 - 6 } & $\sigma_{\psi}=2^{\circ}$ & 9.1620 & 22.7878 & 0.2112 & 0.6065 \\
\hline
\end{tabular}




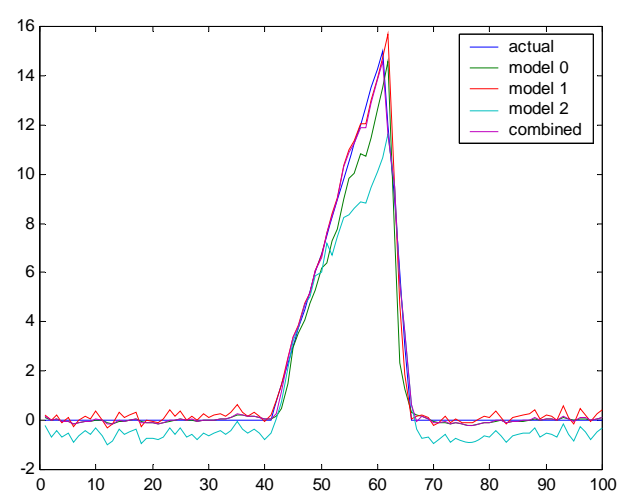

Figure 15. Velocity Errors $\left(\mathrm{V}_{\mathrm{x}}\right)$

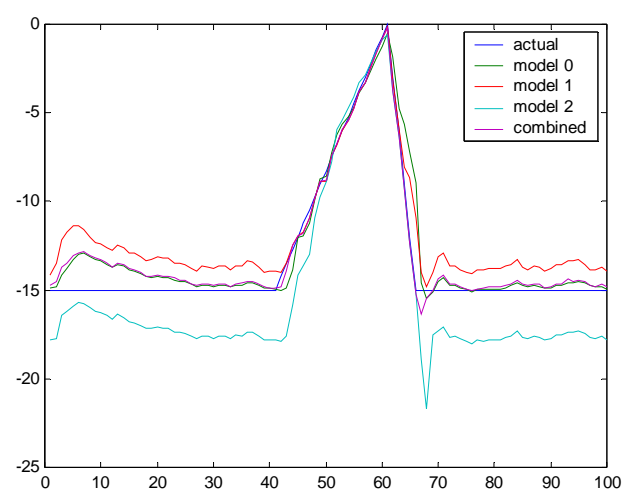

Figure 16. Velocity Errors $\left(\mathrm{V}_{\mathrm{y}}\right)$

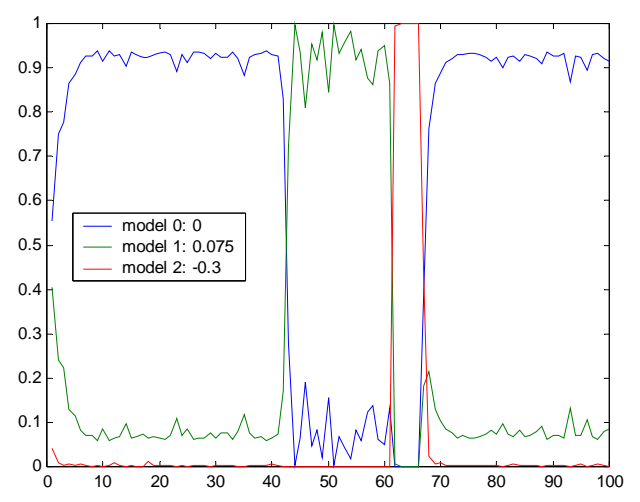

Figure 17. Model Probabilities with Pose Angles

Similar results are shown in Table 2 for the cases where the angular measurement error standard deviation is of $1^{\circ}$ and $2^{\circ}$ while the position measurement error standard deviation is $100 \mathrm{~m}$.

The simulation results in Tables 1 and 2 show that when position and pose angular measurement errors are increased, the target state estimates degrade correspondingly, as one would expect. However, the solution is much better with pose measurements than without, indicating the benefits of feature aiding in maneuvering target tracking.

Table 2. Position and Velocity Estimation Errors Statistics

\begin{tabular}{|c|c|c|c|c|c|}
\hline Pose & STD & RMS-X & RMS-Y & RMS-VX & RMS-VY \\
\hline Without & $\sigma_{x, y}=100 \mathrm{~m}$ & 47.1096 & 62.5025 & 10.1022 & 10.6808 \\
\hline \multirow{2}{*}{ With } & $\sigma_{\psi}=1^{\circ}$ & 9.4412 & 35.3756 & 0.1528 & 0.5203 \\
\cline { 2 - 6 } & $\sigma_{\psi}=2^{\circ}$ & 12.9250 & 38.1557 & 0.2260 & 0.6596 \\
\hline
\end{tabular}

\section{Model Sensitivity of IMM Estimator}

It is well known that the model probability estimates of an IMM estimator are sensitive to such model parameters as the process and measurement noise variances. In this section, we study the sensitivity of the IMM algorithm in terms of its model probabilities with respect to the model acceleration values.

Case 1: $\mathbf{A}_{\text {filter }}=\mathbf{A}_{\text {truth }} / \mathbf{2}$. In this simulation, all the parameters are kept exactly the same as in the previous setting except for the acceleration values used in Model 1 and Model 2, which are halved as compare to the true values, that is, $A_{\text {filter }}=A_{\text {truth }} / 2$.

Figure 18 shows the model probabilities. Compared to Figure 6, the filter is less sure during the quiescent without maneuver since the three models are made closer than the actual data. But it is much better than without pose angular measurements were used.

Figure 18. Model Probabilities with Halved

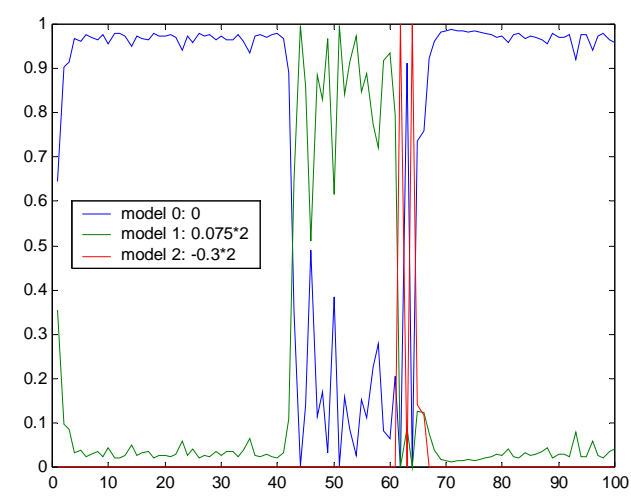

Acceleration

Case 2: $\mathbf{A}_{\text {filter }}=\mathbf{2} \times \mathbf{A}_{\text {truth }}$. In this simulation, all the parameters are kept exactly the same except for the acceleration values used in Model 1 and Model 2, which are doubled as compared to the true values, that is, $A_{\text {filter }}=2 \times A_{\text {truth }}$.

Figure 19 shows the model probabilities when the accelerations used in Model 1 and Model 2 are double of the true values. It shows better model separation during the non-maneuver period than 
Figure 6 since the maneuvering models are made far away from the non-maneuvering model.

However, it becomes more volatile during maneuvers and becomes less sure about the maneuver models because the model values are larger than the actual ones.

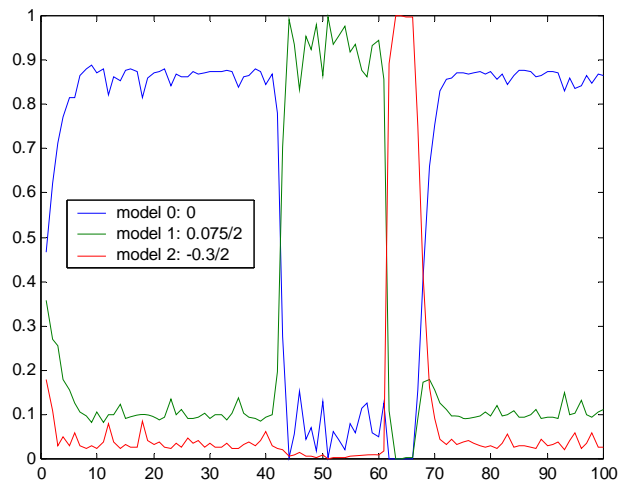

Figure 19. Model Probabilities with Doubled Acceleration

The RMS values are summarized in Table 3, showing corresponding degradation due to filter parameter mismatches.

Table 3. Position and Velocity Estimation Errors Statistics

\begin{tabular}{|c|c|c|c|c|c|}
\hline STD & $\left(\mathbf{A}_{\mathbf{x}}, \mathbf{A}_{\mathbf{y}}\right)$ & RMS-X & RMS-Y & RMS-VX & RMS-VY \\
\hline \multirow{2}{*}{$\begin{array}{c}\sigma_{\mathrm{x}, \mathrm{y}}=50 \mathrm{~m} \\
\sigma_{\psi}=1^{\circ}\end{array}$} & $\div 2$ & 12.9613 & 19.4996 & 0.2124 & 0.3881 \\
\cline { 2 - 6 } & $\times 2$ & 11.9094 & 52.7609 & 0.2463 & 1.1802 \\
\hline
\end{tabular}

\section{Summary}

In this paper, we studied the target pose angular features and their use to improve target tracking. It was based on two physical facts: (1) the longitudinal axis (determined from the pose angles) of a ground target aligns most of the time with the velocity vector and (2) target body rotation is faster than (i.e., preceding to) lateral displacement after a turn maneuver, thus being more easily detectable. We investigated the use of pose angles as a turn maneuver indicator or as an extra measurement to a tracking filter. In the former case, we developed a Bayesian-Markov estimator for turn maneuvers and an IMM estimator for pose angles. In the latter case, we showed significant improvement in position RMS errors with pose angular measurements. We also investigated the effects of pose angular measurement accuracy on estimation performance as well as the sensitivity of the IMM estimator with respect to model parameters.
Since long, target features have been used for classification and ID. Recently it has also been used to facilitate and improve report to track association. This paper has shown that target features can also be used directly in kinematic state estimation, therefore serving as a viable coupling between the target identification and tracking systems.

\section{References}

[1] S.H. Musick, J.U. Sherwood, T.L. Piatt, N.A. Carlson, A Simulation System for Feature-Aided Tracking Research,” SPIE Defense \& Security Symp., April 2004

[2] C. Yang and E.P. Blasch, "Mutual-Aided Target Tracking and Identification,” SPIE AeroSense, April 2003.

[3] E. Blasch and C. Yang, "Ten Methods to Fuse GMTI and HRRR Measurements for Joint Tracking and Identification,” Fusion 2004, July 2004.

[4] J.R. Layne and D. Simon, A Multiple Model Estimator for A Tightly-Coupled HRR Automatic Target Recognition and MTI Tracking System, SPIE Conf. on Algorithms for Synthetic Aperture Radar Imagery, 1999.

[5] H.A.P. Blom and Y. Bar-Shalom, Interacting Multiple Model Algorithm for Systems with Markov Switching Coefficients, IEEE Trans. Automatic Control, 33(8), 1988.

[6] C. Yang, Y. Bar-Shalom, and C.F. Lin, Maneuvering Target Tracking with Image-Enhanced Measurements, Proc. of American Control Conference, 1991.

[7] C. Yang, Y. Bar-Shalom, and C.F. Lin, Discrete-Time Point Process Filter for Mode Estimation, IEEE Trans. on Automatic Control, 1992.

[8] E. Blasch, Derivation of A Belief Filter for High Range Resolution Radar Simultaneous Target Tracking and Identification, Ph.D. Dissertation, Wright State University, 1999. 